\title{
HIPERTENSÃO RENOVASCULAR LEVANDO À NEFRECTOMIA UNILATERAL: RELATO DE CASO
}

\author{
RENOVASCULAR HYPERTENSION LEADING TO UNILATERAL NEPHRECTOMY: \\ CASE REPORT
}

Renan Santiago Faria ${ }^{1,2}$, Bianca Moreira de Aquino ${ }^{3,4}$, Astria Mariana Brito de Souza ${ }^{5}$, Fernanda Caroline Silva ${ }^{6}$, Laura Amaral Boaventura ${ }^{6}$, Luciana dos Santos Pinto ${ }^{7}$, Erika Maria Gonçalves Campana $8,9,10$

\section{RESUMO}

Objetivo: Relatar o caso de uma paciente jovem portadora de Hipertensão Arterial Secundária à estenose da artéria renal, que evoluiu com perda renal em decorrência de necessidade de nefrectomia unilateral, enfatizado a importância do diagnóstico e da abordagem adequada desta patologia para o controle da pressão arterial e preservação da função renal. Método: Os dados foram obtidos através de entrevista com a paciente, análise de prontuário, laudos de técnicas diagnósticas, as quais a paciente foi submetida, entre julho e novembro de 2019, durante as consultas médicas e revisão bibliográfica. Conclusão: A nefrectomia unilateral mostrou-se eficaz no controle da hipertensão arterial, na melhora do desempenho renal, possibilitando a melhoria na qualidade de vida do indivíduo afetado.

Descritores: Hipertensão Arterial Sistêmica Secundária; Hipertensão Renovascular; Nefrectomia Unilateral.

\begin{abstract}
Objective: To report the case of a young patient with SAH secondary to renal artery stenosis, who developed renal loss due to the need for unilateral nephrectomy, emphasizing the importance of the diagnosis and the appropriate approach of this pathology for the control of blood pressure and preservation of renal function. Method: The data were obtained through interview with the patient, analysis of medical records, reports of diagnostic techniques, which the patient was submitted between July and November 2019, during medical consultations and literature review. Conclusion: Unilateral Nephrectomy proved to be effective in controlling arterial hypertension, improving renal performance and in the evolution of renal insufficiency that is difficult to control from the renovascular root, enabling improvement in the affected individual's quality of life.
\end{abstract}

Keywords: Secondary Systemic Arterial Hypertension; Renovascular Hypertension; Unilateral Nephrectomy.

\section{INTRODUÇÃO}

A Hipertensão Arterial (HA) é o principal fator de risco para a incidência de acidente vascular cerebral, infarto agudo do miocárdio, insuficiência renal crônica e óbito. ${ }^{1}$ A hipertensão arterial sistêmica secundária (HAS-S) é um tipo de hipertensão arterial ligada a uma patologia potencialmente tratável e de causa identificável, que afeta cerca de 10\% dos hipertensos, mas, que requer um diagnóstico precoce e um tratamento específico. ${ }^{2}$ Entre as causas de HAS secundária estão: a doença renovascular por aterosclerose, associada à estenose parcial ou total, uni ou bilateral renal, variando de $1 \%$ a $5 \%$ na população de hipertensos, desencadeada e conservada por isquemia do tecido renal; o hiperaldosteronismo primário (HAP), devido às alterações de padrões de rastreamento e; a apneia obstrutiva do sono, diante do excesso de peso. ${ }^{3}$

A estenose ou oclusão de uma artéria renal pode ocasionar hipertensão pela estimulação da liberação da renina das células justaglomerulares do rim afetado. Cabe desconfiar da

1. Centro de Terapia Intensiva da Santa Casa de Diamantina,M, Brasil.

2. Setor de Clínica Médica do Hospital Nossa Senhora da Saúde de Diamantina, MG, Brasil.

3. Programa de saúde da família, Universidade Gama Filho, RJ, Brasil.4. Estratégia de saúde da família em CF Wilma Costa/RJ, Brasil.

5. Universidade iguaçu-campus Nova Iguaçu, RJ, Brasil.

6. Faculdade de Medicina da Universidade Federal dos Vales do Jequitinhonha e Mucuri - Campus Jk, MG, Brasil.

7. Universidade Unigranrio - Caxias, RJ,Brasil.

8. Ambulatório de Hipertensão e Lípides no Departamento Cardiopulmonar da UERJ, RJ,Brasil.

9. Faculdade de Medicina da Universidade Iguaçu, RJ, Brasil.

10. Núcleo Especializado de Hipertensão na Clínica SEACOR, Diretora Administrativa do Departamento de Hipertensão da SBC (2O2O-21).

Correspondência: Erika Campana. Rua São Januário, no 159/1103 B. Fonseca - Niteroi/RJ - CEP: 24130-386. unigpesqmed@gmail.com 
presença de hipertensão renovascular se a hipertensão surgir em pacientes abaixo de 20 ou acima dos 50 anos, se houver agravamento do estadiamento da hipertensão previamente diagnosticada sem outro fator relacionado que possa explicar o ocorrido, nos casos de hipertensão de estagio três, especialmente nos casos resistentes à terapêutica medimentosa. ${ }^{4}$

A HAS renovascular é um fator de risco para doenças cardiovasculares (angina inconstante, infarto agudo do miocárdio e insuficiência cardíaca) e cerebrovasculares (acidentes vasculares cerebrais). ${ }^{5}$ Sendo, ainda, a principal causa de HAS maligna (nefrosangiosclerose maligna), altamente letal, podendo acarretar oclusão do lúmen do vaso e consequente diminuição do curso sanguíneo renal; ou refratária com insuficiência renal progressiva, assimetria de tamanho ou função renal, resultando em isquemia renal, complicações severas e óbito. ${ }^{6}$ Cabe enfatizar que um a maioria dos pacientes hipertensos também apresenta outras comorbidades como: diabetes e dislipidemia. Deste modo, o diagnóstico precoce e o tratamento apropriado são imprescindíveis.

\section{OBJETIVO}

Relatar o caso de uma paciente jovem portadora de HAS-S a estenose de arterial renal que evoluiu com perda renal em decorrência de necessidade de nefrectomia unilateral, enfatizado a importância do diagnóstico e da abordagem adequada desta patologia para o controle da pressão arterial e preservação da função renal.

\section{MÉTODO}

Trata-se de um estudo de caso clínico. Os dados contidos neste artigo foram obtidos através de entrevista com a paciente, análise de prontuário, laudos de técnicas diagnósticas, as quais a paciente foi submetida, entre julho e novembro de 2019, em uma Clínica Cardiológica, situada em Diamantina, MG, durante as consultas médicas e revisão bibliográfica. A paciente forneceu consentimento para a publicação dos seus dados clínicos, com a preservação da sua identidade.

\section{CASO CLÍNICO}

Anamnese: Paciente D.C.S., sexo feminino, 32 anos, etilista social, sedentária, sobrepeso, sem controle dietético. Pré-eclâmpsia há cerca de seis anos e histórico de trauma abdominal após queda de moto, com hemoperitôneo, devido à lesão hepática grau II, tratado com hepatorrafia. Sem outras comorbidades. Negava alergias e tabagismo.

Diagnosticada com HA estágio II após períodos de cefaleia esporádica, devido ao paroxismo dos sintomas não procurou atendimento médico e não utilizou anti-hipertensivo. Em março de 2019 apresentou novos períodos de crise hipertensiva, com necessidade de ida ao pronto atendimento, mesmo sem nenhum episódio de emergência ou urgência hipertensivas. Devido ao aumento dos níveis pressóricos e persistência dos sintomas, procurou consultório cardiológico. Encaminhada pelo plantonista do pronto atendimento como quadro de enxaqueca associado a aumento dos níveis pressóricos pela dor. Estava em uso de losartana, prescrito pelo médico da emergência.

\section{Queixa principal: Crise hipertensiva}

Em consulta cardiológica, foi iniciada a investigação de HAS-S, devido à idade da paciente e por ser hipertensão estágio II, assim foi modificado o esquema terapêutico para indapamida e anlodipino, por ser mulher em idade fértil.

Exame físico: Bom estado geral, corada, hidratada, anictérica, acianótica, afebril, lúcida e orientada em tempo e espaço. Pressão arterial MSE $170 \times 100$ mmHg 2 aferições, Pressão arterial MSD 160 x 100 mmHg. Os pulsos carotídeos e os membros superiores encontravam-se simétricos, sem sopros e sem clicks, bulhas normofonéticas, ritmo cardíaco regular em dois tempos. Aparelho respiratório: murmúrio vesicular sem ruídos adventícios. Sem distensão abdominal, ruídos hidroaéreos presentes, sem visceromegalias, sem massas na palpação, sem sopros na auscuta. Sem déficit focal, orientada, sem alterações em marcha, pupilas isocóricas, escala de coma de Glasgow 15.

Durante a realização dos exames, a paciente referiu náuseas e mal estar e atribuiu que seriam efeitos colaterais do indapamida, com isso foi trocada a medicação para clortalidona e anlodipino. Em avaliação dos exames foi evidenciado hipocalemia em uso de diurético tiazídico associado à proteinúria em nível próximo ao superior da normalidade. Retirado clortalidona, realizado reposição de potássio, iniciado olmesartana e mantido anlodipino. Apresentou hipotensão após início de bloqueador de receptor de angiotensina (BRA), sendo então retirado o anlodipino. Após ajuste, o tratamento foi guiado por meta terapêutica e definido pela estratificação de risco do paciente. Devido à hipocalemia, podendo ser induzida por diurético ou espontânea com piora pelo diurético e hipotensão no início de BRA. Iniciada investigação de hiperaldosteronismo primário e hipertensão renovascular. Hipótese diagnóstica: Hipertensão arterial de difícil controle. Suspeita de HAS-S em paciente jovem. Hiperaldosteronismo primário? Hipertensão renovascular?

\section{Investigação complementar}

Exames laboratoriais (23/07/2019): solicitados, identificou-se alterações nas funções renais, com valores acima dos limites considerados normais (Tabela 1).

Ultrasonografia renal e doppler de artérias renais (18/06/2019): dimensões reduzidas do rim direito (hipotrofia renal direita) e litíase renal esquerda. Artéria renal direita não visualizada. Artéria renal esquerda: pico de velocidade sistólica: $36,6 \mathrm{~cm}$. Aorta-abdominal: $47 \mathrm{~cm} / \mathrm{s}$. (Figura 1)

Em 11/07/19: PAM 132/91 mmhg. Vigília: 136/95 mmhg.

Tabela 1. Exames laboratoriais solicitados.

\begin{tabular}{c|c|c|c|c|c|c|c} 
& Aldosterona & Cortisol & Estradiol E2 & FSH & LH & Insulina & Renina \\
\hline $23 / 07 / 2019$ & $19,1 \mathrm{ng} / \mathrm{dL}$ & $10,8 \mathrm{ug} / \mathrm{dL}$ & $63 \mathrm{pg} / \mathrm{mL}$ & $6,33 \mathrm{mUL} / \mathrm{mL}$ & $13 \mathrm{mUL} / \mathrm{mL}$ & $21 \mu \mathrm{UL} / \mathrm{mL}$ & $71 \mu \mathrm{UI} / \mathrm{mL}$ \\
\hline & $\mathrm{K}+$ & $\mathrm{Na}+$ & - & - & - & - & - \\
\hline $25 / 07 / 2019$ & $4,1 \mathrm{mE} / \mathrm{qL}$ & $138 \mathrm{mE} / \mathrm{qL}$ & & & & & \\
\hline
\end{tabular}




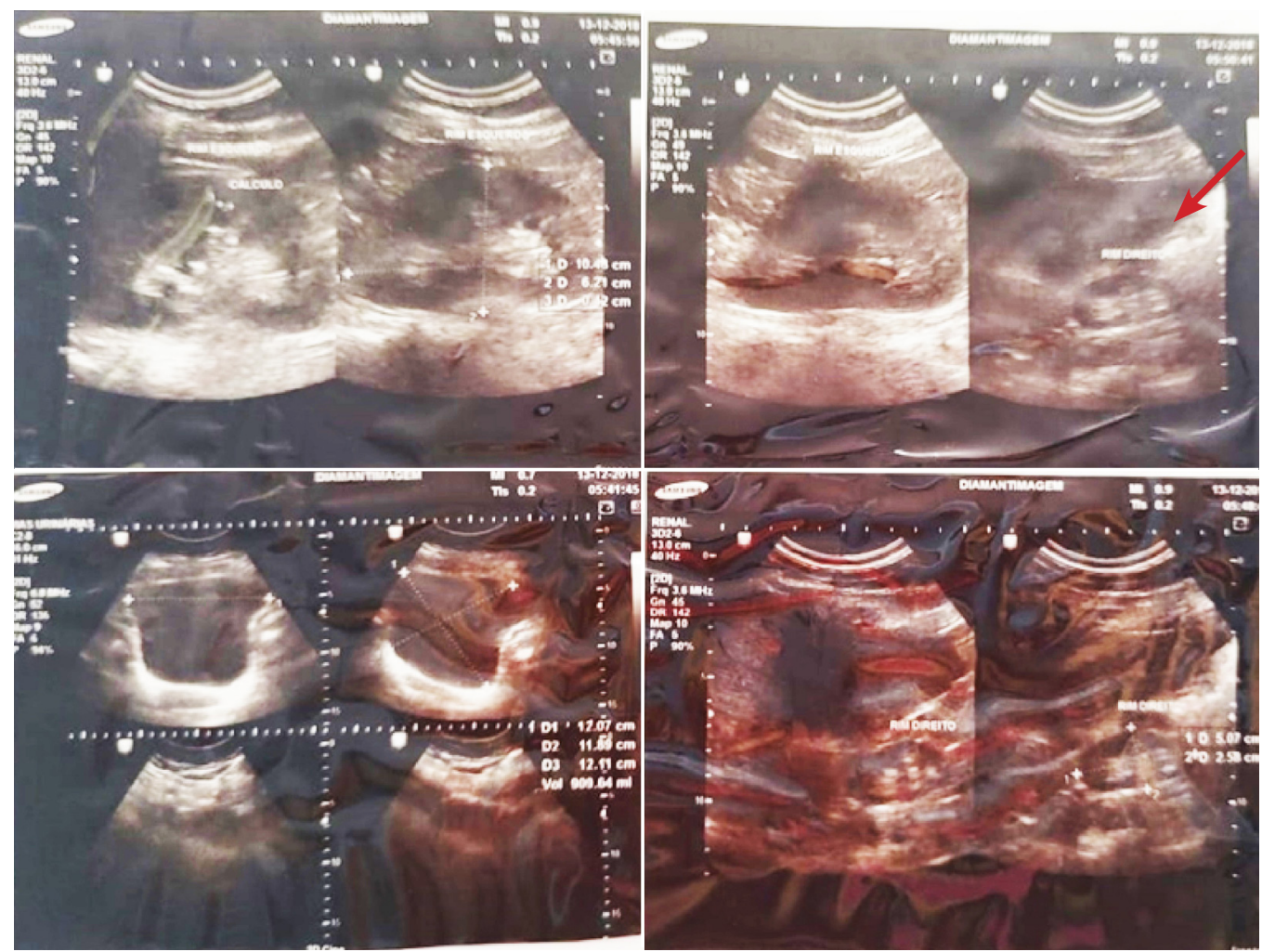

Figura 1. Ultrassonografia Renal evidenciando a hipertrofia renal direita.

Angiotomografia abdominal (12/07/2019): estreitamento difuso da artéria renal associado a alterações perfusionais com exclusão funcional e atrofia renal direita. Artéria e veia renal esquerda sem alterações.

Tomografia computadorizada de abdome e pelve (23/08/2019): estudo tomográfico Multislice do abdome superior, sinais de nefropatia crônica à direita. No restante do exame não foram detectáveis pelo método, alterações importantes.

Devido à renina elevada, foi solicitado cintilografia renal (24/08/2019), evidenciando rim esquerdo de aspecto vicariante, sem anormalidades. Exclusão funcional do Rim direito. E a Cintilografia Renal Dinâmica com Radiofármaco: DTPA-Tc 99m Dose: 10 mCi, evidenciou função glomerular e vias excretoras pérvias preservadas à esquerda. Ausência de perfusão, captação, concentração e excreção importantes do radiofármaco pelo rim direito, sendo o padrão cintilográfico compatível com exclusão funcional (ausência de função).

\section{CONDUTA}

Devido à permanência da Renina elevada, possivelmente por resíduos de tecido com sofrimento isquêmico, foi indicada Nefrectomia direita e biopsia. Após cirurgia, a paciente evoluiu com melhora dos níveis pressóricos, tolerando a suspensão à medicação, guiada através do MAPA. Em retorno de consultório dia 09/10/2019, a biopsia renal revelou achados de hipertrofia renal e nefrite túbulo intersticial, compatível com alteração isquêmica. Artéria renal sem sinais de displasia no segmento enviado, com estritamente discreto e difuso.
Diante da ausência de displasia no segmento avaliado foram solicitados marcadores sorológicos de vasculite, com a finalidade de encontrar explicações para o estreitamento da artéria renal. Em 14/11/2019, a paciente retornou ao consultório para a revisão laboratorial evidenciando: complemento C3 139 / C4: 22 P Anca Reagente. FAN: 1:160/ Nuclear pontilhado fino denso Placa metafásica reagente, núcleo reagente. Em 06/08/2020, na revisão de lâmina, confirmou-se a descrição dos achados e a interpretação diagnóstica, no contexto de estenose da artéria renal, conforme informação clínica. Não identificado sinais de vasculite na porção de tecido enviada.

\section{DIAGNÓSTICO DEFINITIVO E RESOLUÇÃO DO QUADRO}

HAS-S de causa renovascular, tratada com nefrectomia unilateral direita, apresentando sucesso para cura da HA. A paciente permanece em acompanhamento com interconsulta de reumatologista para diagnóstico de vasculite diante da sorologia positiva, podendo ser a possível causa da estenose da artéria renal. Mantém ausência de alterações pressóricas.

\section{DISCUSSÃO}

A HAS-S causada por doença renal decorre da diminuição da perfusão renal, isquemia renal e subsequente estimulação do sistema renina-angiotensina-aldosterona (SRAA) do rim comprometido. A angiotensina II ativa, provoca vasoconstrição e a produção excessiva de aldosterona, resulta 
em retenção de sódio e líquidos, promovendo elevação da pressão arterial sustentada.?

A paciente do caso trata-se de uma mulher jovem de 32 anos, com HA evoluindo com perda renal unilateral direita, o que estabelece a importância de considerar causas secundárias. Nos exames clínicos não havia quaisquer sinais ou sintoma que sugerissem a possível causa, portanto foi imprescindível averiguar as causas endócrinas, onde o hiperaldosternismo é uma das mais prevalentes (9\% a $37 \%$ dos casos) ${ }_{i}^{8}$ e causas não endócrinas, onde a origem renovascular em uma mulher jovem deve ser avaliada.

Inicialmente os sinais clínicos e os exames laboratoriais (Tabela 1), sugeriam a probabilidade de aldosteronismo primário ${ }^{8}$ (Hipocalemia importante induzida pelo uso de diurético, $\mathrm{K}+2,9 \mathrm{mEq} / \mathrm{L})$; entretanto os níveis de renina elevados $(71 \mu \mathrm{Ul} / \mathrm{mL})$ não eram compatíveis com este diagnóstico. Todavia, os demais resultados dos exames para causas endócrinas descartaram o aldosteronismo primário (relação aldosterona/ atividade plasmática de renina e a presença de renina alta que não eram compatível com o hiperaldosteronismo). Neste caso, com o rim esquerdo normal, e o SRAA superativado pelo rim direito isquêmico, esta ativação poderia explicar a renina elevada e a hipocalemia induzida pelo uso de diurético.

Excluídas as causas endócrinas, a causa renovascular foi um diagnóstico pensado para este caso. A prevalência da HA renovascular é estimada entre $1 \%$ e $5 \%$ da população geral de hipertensos com idade entre 20 e 50 anos. Em pacientes mais idosos com hipertensão refratária ou acelerada, estes valores podem alcançar de 15\% a 45\%. A realização da ultrassonografia com doppler foi o exame de rastreamento para se determinar o diagnóstico. ${ }^{5-7}$

Assim, as análises dos resultados da ultrassonografia de artérias renais e a angiotomografia abdominal, revelaram achados anatômicos e perfusionais compatíveis exclusão funcional e atrofia renal direita. (Figura 1 e 2)

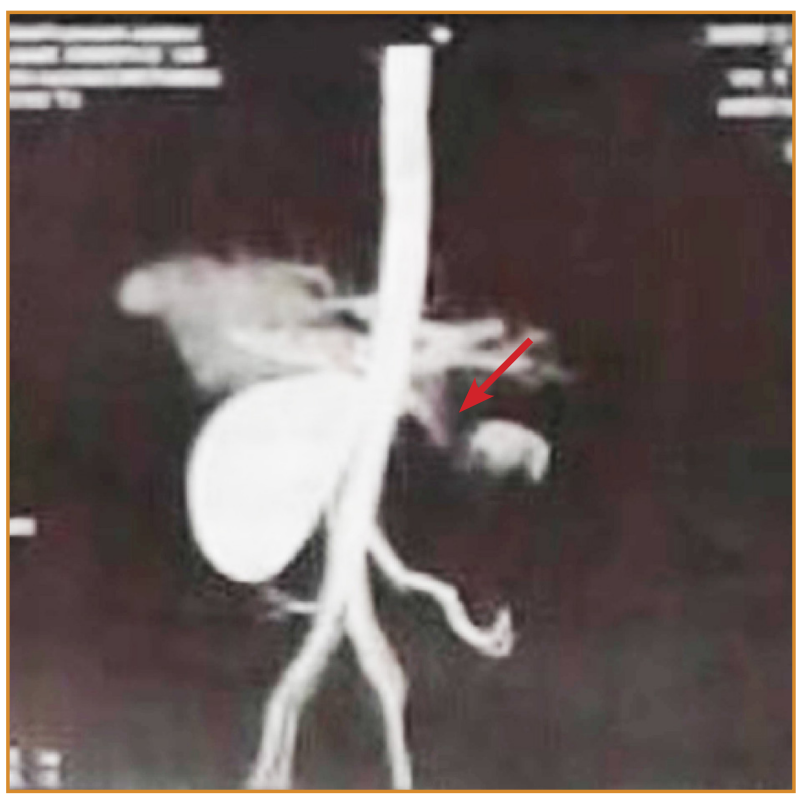

Figura 2. Angiotomografia abdominal evidenciando a estenose da artéria renal direita.
Por fim, a cintilografia renal proporcionou informação sobre a dimensão renal, perfusão e capacidade excretória, portanto, confirmou a exclusão funcional do rim direito.

A indicação de nefrectomia na paciente deste caso se justifica pela presença de hipertensão de difícil controle e pela evidência de isquemia renal. A realização de nefrectomia curativa em casos de HA renovascular é uma opção nos casos de obstrução total da artéria renal com rins menores do que $8 \mathrm{~cm}$, sobretudo quando existe produção aumentada de renina pelo rim afetado. A nefrectomia de rins atróficos proporciona a melhora do controle da pressão arterial em $70 \%$ dos pacientes, restabelecendo o fluxo sanguíneo renal apropriado, melhorando o desempenho renal em mais de $60 \%$. ${ }^{7,9}$ Neste sentido, o objetivo da nefrectomia direita era o controle pressórico e preservação do rim esquerdo.

A presença de proteinúria significativa, circulação cortical pobre, e o comprometimento com oclusão total das artérias renais, são fatores que sugerem pouca probabilidade de reversão da nefropatia isquêmica crônica, achados evidenciados nos exames de imagem desta paciente (rim de pequeno tamanho, disfuncionante), sendo portanto elementos que colocam a revascularização ou angioplastia da artéria renal como opção não indicadas neste caso. ${ }^{7,9}$

Um estudo realizado em 51 pacientes com hipertensão renovascular, portadores de rim atrófico por estenose, submetidos à nefrectomia no período de 1989 a 2007, revelou que 49 pacientes (69\% entre 34 a 49 anos) obtiveram benefícios com o procedimento do ponto de vista pressórico e uma melhora no desempenho renal em 63,8\% após um ano, com uma taxa de mortalidade de $2 \%$, apresentando cura ou melhora da pressão arterial ou mínima necessidade de medicação hipotensora. ${ }^{10} \mathrm{Na}$ paciente em questão, a realização da nefrectomia resultou em cura da HÁ com possibilidade de retirada de todos os fármacos anti-hipertensivos.

Enfim, a HA renovascular, pode resultar em alteração da pressão de perfusão e do fluxo sanguíneo renal, isquemia renal e consequentemente, na perda da função renal. Este quadro clínico pode se reversível quando detectado precocemente, possibilitando o controle da pressão arterial e a preservação da função renal, mas quando feito tardiamente, pode levar à necessidade de nefrectomia. Portanto, o adequado reconhecimento das causas secundárias de HÁ em pacientes jovens é fundamental para o controle pressórico e também para proteção dos órgãos-alvo, diminuindo a morbimortalidade cardiovascular. ${ }^{5}$

\section{CONCLUSÂO}

O caso relatado e a revisão da literatura realizada emergem o debate sobre o tratamento de uma condição importante e difícil que é a HA renovascular e corrobora o elevado impacto negativo à qualidade de vida do indivíduo. O reconhecimento precoce dos hipertensos, a identificação das causas possivelmente reversíveis e a terapêutica eficaz adequada, são imprescindíveis. A opção de abordagem nesse caso foi a nefrectomia unilateral, por permitir tanto o controle pressórico, quanto a preservação do rim viável contralateral. 


\section{REFERÊNCIAS}

1. Malta DC, Gonçalves RPF, Machado IE, Freitas MIF, Azeredo C, Szwarcwald CL. Prevalência da hipertensão arterial segundo diferentes critérios diagnósticos, Pesquisa Nacional de Saúde. Rev. bras. epidemiol. 2018;21( supl.1). Epub Nov 29, 2018. doi: 10.1590/1980-549720180021.supl.1.

2. Dinis PG, Cachulo, MC, Fernandes A, Paiva L, Gonçalves L. Hipertensão Arterial Sistémica Secundária: Incertezas do Diagnóstico. Acta Med Port; 2017; ;30(6):493-6. doi: 10.20344/amp.8007.

3. Pereira AA, Cordeiro Jr. AC, Nogueira A, et al. Hipertensão Arterial Sistêmica Secundária. J. Bras. Nefrol. 2010:32( supl.1) doi: 10.1590/S010128002010000500009.

4. Bakris GL. Hipertensão renovascular. Manuais MSD edição para profissionais de saúde. Acesso em: Fev. 2018. Disponível em: https://www.msdmanuals. $\mathrm{com} / \mathrm{pt} /$ profissional/doen $\% \mathrm{C} 3 \% \mathrm{~A} 7 \mathrm{as}-$ cardiovasculares/hipertens\%C3\%A3o/ hipertens\%C3\%A3o-renovascular

5. Rodrigues MS. Hipertensão renovascular: A importância da sua identificação clínica na abordagem da hipertensão arterial sistêmica (HAS). Rev. Med
Minas Gerais. 2019;29: e-2038. doi: 10.5935/2238-3182.20190055.

6. Vilela-Martin JF, Yugar-Toledo JC, Rodrigues MC, et al. Posicionamento Luso-Brasileiro de Emergências Hipertensivas - 2020. Arq Bras Cardiol. 2020;114(4)736-51.

7. Bortolotto LA, Malachias MVB. Atualização no diagnóstico e tratamento das principais causas de hipertensão secundária. Rev Bras Hipertens. 2011:18(2):46-66.

8. Malachias MVB, Souza WKSB, Plavnik FL, et al. $7^{\text {a }}$ Diretriz Brasileira de Hipertensão Arterial. Arq Bras Cardiol 2016; 107(3Supl.3):1-83.

9. Silva FEM. Acometimento da função renal nos portadores de hipertensão arterial sistêmica - revisão de literatura. (Monografia de Pós-graduação em Atenção Básica em Saúde da Família) - Universidade Federal de Minas Gerais - UFMG, Lagoa Santa - MG, 2013.

10. Thomaz MJ. Papel da nefrectomia do rim atrófico nos portadores de hipertensão renovascular. (Dissertação de Mestrado em Urologia) Faculdade de Medicina da Universidade de São Paulo - USP, 2008. 